\title{
New hope for patients and challenges for the multidisciplinary arrhythmia team: a hybrid convergent approach for atrial fibrillation treatment
}

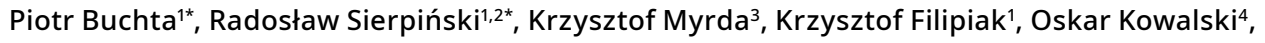 \\ Witold Bratkowski ${ }^{5}$, Zbigniew Kalarus', Mariusz Gąsior ${ }^{3}$, Michał O. Zembala ${ }^{5}$ \\ 1 Silesian Center for Heart Diseases, Zabrze, Poland \\ 2 Collegium Medicum, Cardinal Wyszynski University in Warsaw, Institute of Cardiology in Anin, Warsaw, Poland \\ 3 Silesian Center for Heart Diseases, 3rd Department of Cardiology, SMDZ in Zabrze, Medical University of Silesia, Katowice, Zabrze, Poland \\ 4 Department of Dietetics, Faculty of Public Health in Bytom, Medical University of Silesia in Katowice, Silesian Centre of Heart Disease in Zabrze, I Department of Cardiology \\ 5 Silesian Center for Heart Diseases, Department of Cardiac, Vascular and Endovascular Surgery and Transplantology, Medical University of Zabrze, Poland \\ 6 Silesian Center for Heart Diseases, Department of Cardiology, Congenital Heart Diseases and Electrotherapy, Zabrze, Poland
}

\section{KEY WORDS}

atrial fibrillation, convergent ablation, hybrid ablation, minimally invasive transabdominal approach

\footnotetext{
Correspondence to: Michał 0. Zembala, MD, PhD, Silesian Center for Heart Diseases, Department of Cardiac, Vascular and Endovascular Surgery and Transplantology, Medical University of Zabrze, ul. M. Curie-Skłodowskiej 9 41-800 Zabrze, Poland, phone: +48324793466, email: m.zembala.jr@sccs.pl Received: August 13, 2020. Revision accepted: October 2, 2020. Published online: October 6, 2020. Kardiol Pol. 2020; 78 (12): 1243-1253 doi:10.33963/KP.15640 Copyright by the Authors(s), 2020

* PB and RS contributed to this study equally.
}

\section{ABSTRACT}

BACKGROUND Available data suggest the important role of ablation of the left atrial posterior wall and epicardial myocardial layers in rhythm control therapy in patients with persistent drug-refractory atrial fibrillation (AF). However, endocardial ablation is not always effective in transmural substrate modification. The alternative treatment option is minimally invasive hybrid approach (HABL) combining the strengths of surgical and catheter ablation.

AIMS This study aimed to assess the periprocedural safety as well as acute and long-term outcomes of HABL for AF.

METHODS This is a retrospective single-center study of patients who underwent HABL using the minimally invasive transabdominal approach between July 2009 and January 2020. Demographic in-hospital data and 12-month follow-up results were obtained. The number of hospitalizations, cardioversions, re-ablations, and severe adverse events in a 3-year period before and after HABL were compared using data from the national healthcare provider.

RESULTS In total, 158 patients (mean [SD] age, 51.02 [10.67] years) who underwent HABL were included; $61.4 \%$ had persistent AF. There was a $4.4 \%$ incidence of periprocedural complications without any fatalities. In $66 \%$ of patients, additional endocardial substrate modification was needed, in $52.6 \%$ on the posterior wall. In the 12-month follow-up, most patients (78.3\%) remained free of arrhythmias. There was a significant reduction in the number of hospitalizations (for $A F, 1.65$ vs 0.54 ; or any other cause, 2.56 vs 1.31 per patient), cardioversions, and re-ablations after $\mathrm{HABL}$ (all $P<0.05$ ).

CONCLUSIONS The hybrid multidisciplinary approach for treatment of AF is a safe and very effective treatment method in long-term follow-up, which reduces healthcare burden. It could be considered as an alternative therapeutic option especially in patients with persistent AF.

INTRODUCTION The most frequent arrhythmia in developed countries is atrial fibrillation (AF). The prevalence of AF increases with age and presence of comorbidities, especially heart failure or mitral valve diseases. Due to the progressive aging of society, a proportional increase in the number of patients with AF should be expected. Atrial fibrillation not only worsens 


\section{WHAT'S NEW?}

This is the first study that shows the comprehensive assessment of the perioperative course of hybrid ablation for atrial fibrillation, including a detailed analysis of extension of endocardial ablation needed for patient-tailored therapy during the second stage of treatment, and the assessment of long-term outcomes. We also analyzed the impact of hybrid ablation therapy on the number and type of medical contacts in a 3-year period before and after treatment based on national healthcare provider records. We show a substantial reduction in healthcare burden following hybrid ablation for atrial fibrillation. isolation, which, taking into account the pathophysiology of persistent $\mathrm{AF}$ and the presence of arrhythmogenic substrate in other areas of the atria, may limit the long-term results. Additionally, leaving the areas of slow-conducting tissue may promote the induction and persistence of macro-reentry tachycardias.

The hybrid (convergent) procedure was developed to combine the strengths of surgical and endocardial catheter ablation into a single procedure. A cumulative analysis of patients undergoing hybrid ablation showed no recurrence of arrhythmia in about $75 \%$ to $85 \%$ of patients in 1-year follow-up, and a tendency for a persistent effect in a 3-year follow-up, even among those with persistent arrhythmia. However, the results vary depending on the strategy adopted and the experience of the center. ${ }^{16-20}$

To the best of our knowledge, there is no data summarizing the scope of necessary substrate modifications in the second stage of AF treatment with the hybrid transabdominal ablation approach (hybrid ablation, HABL) and HABL effect on the frequency of hospitalizations, cardioversions, and serious cardiovascular events in a long-term follow-up. Therefore, this study aimed to assess the periprocedural safety as well as the acute and long-term outcomes of HABL for AF.

nation of arrhythmias and the maintenance of sinus rhythm, hence, their inhibition may have a decisive impact on further clinical course. ${ }^{11,12}$ Thus, permanent restoration of the sinus rhythm is the goal in symptomatic patients. Unfortunately, pharmacological treatment is (in most cases) ineffective, and percutaneous ablation is currently the most effective treatment option. However, in some patients, the recurrence of $\mathrm{AF}$ is observed, requiring further intensification of treatment, including repeated ablations. A major limitation of the percutaneous ablation method is the possible incomplete isolation of pulmonary veins (PVs) in long-term follow-up, which mainly arises from inefficient delivery of energy to tissue and anatomical conditions, resulting in nontransmural lesions and leaving the substrate for arrhythmia recurrence..$^{13}$ According to the available reports, almost $40 \%$ of ablation lines performed endocardially may require epicardial supplementation to achieve transmurality, even with endocardially confirmed local conduction block. ${ }^{14,15}$

Current recommendations on invasive AF allow a surgical strategy in the case of resistance to antiarrhythmic drug therapy or failed ablations in patients with persistent and persistent long-term $\mathrm{AF}^{3}{ }^{3}$ However, the surgical approach has its limitations, namely, lack of access to all atrial walls and inability to create an electrical map to define the arrhythmia substrate for tailored ablation. Moreover, there are no uniform recommendations for performing percutaneous and surgical ablation, apart from the required PVs
METHODS This is a retrospective analysis of 158 patients who underwent the hybrid (convergent) procedure for AF treatment between July 2009 and January 2020 at the Silesian Centre for Heart Diseases in Zabrze.

Detailed study protocols, surgical and percutaneous CARTO procedures, and preliminary results were published previously. ${ }^{21-23}$ All patients were symptomatic and had failed at least one antiarrhythmic drug therapy. Patients with a previous open-chest surgery or history of complicated percutaneous $\mathrm{PV}$ isolation procedure (perforation, tamponade, $\mathrm{PV}$ stenosis $>50 \%$ ) were not eligible to undergo the hybrid procedure. Before each step of ablation, patients underwent transesophageal echocardiography to exclude intracardiac thrombi. The appropriate approvals of the institutional review board and ethics committee were obtained for the study prior to initiating patient enrolment and all patients provided written informed consent before procedures.

Minimally invasive, surgical ablation protocol Patients were anesthetized using the total intravenous anesthesia technique together with short-acting muscle relaxants. Two independent temperature probes were inserted into the esophagus. A midline abdominal 2-cm incision was made $3 \mathrm{~cm}$ below the xiphoid, through which a 10-mm laparoscopic port was inserted. Once the peritoneum was accessed, $\mathrm{CO}_{2}$ 
A

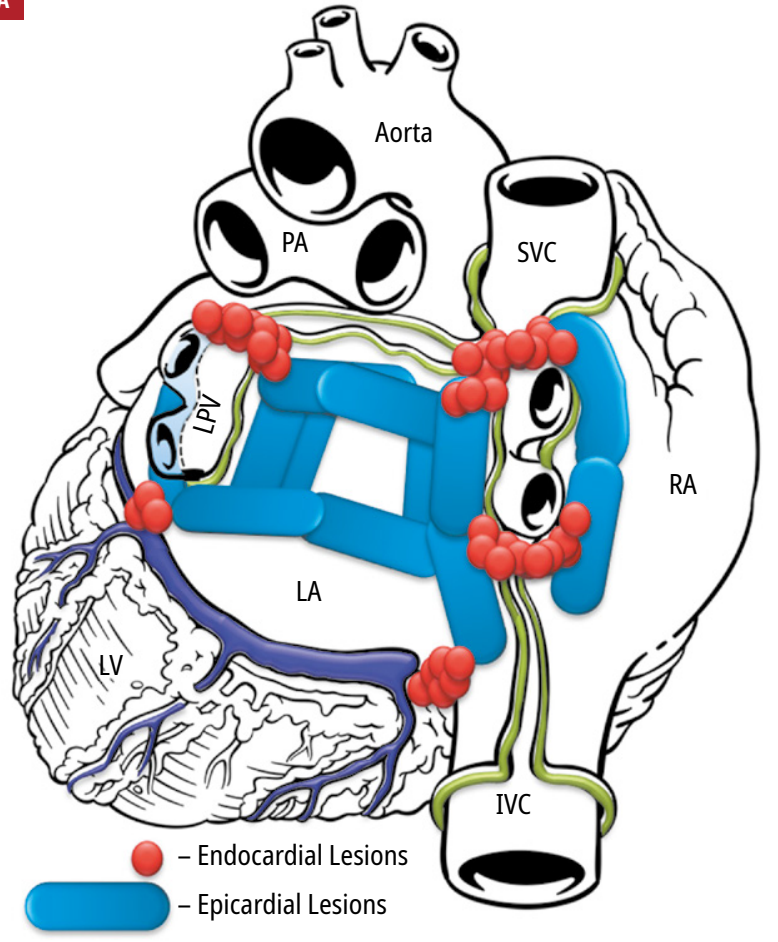

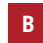

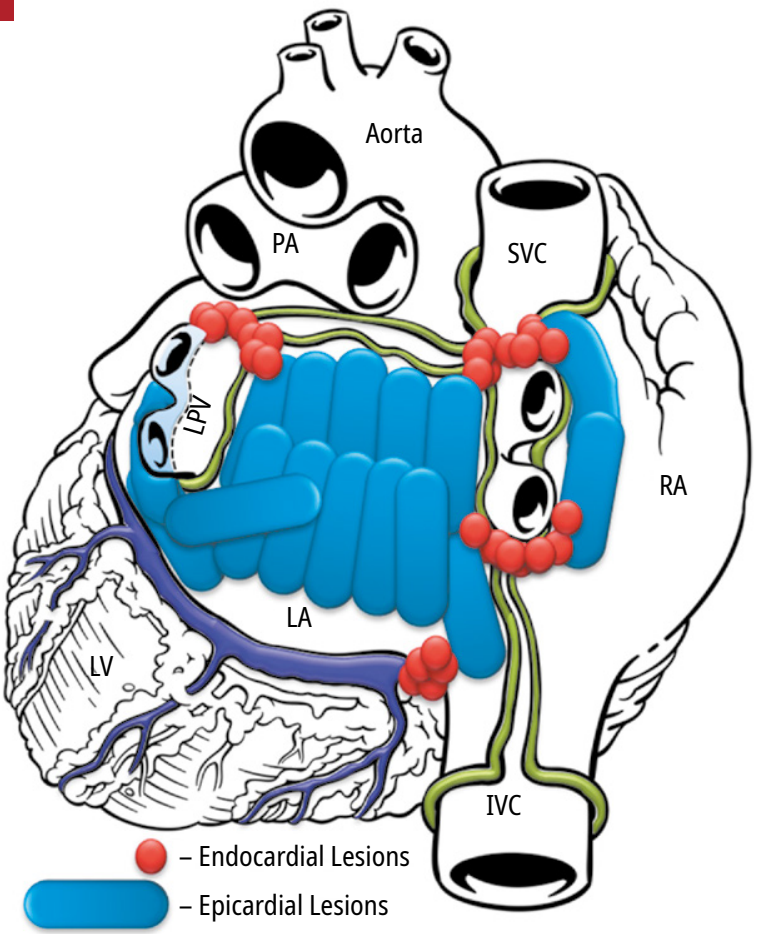

FIGURE 1 Lesion pattern created mostly in patients with paroxysmal and persistent (A) and long-standing persistent (B) atrial fibrillation (adapted with permission from Wojtaszczyk et a ${ }^{33}$ )

Abbreviations: IVC, inferior vena cava; LA, left atrium; PA, pulmonary artery; SVC, superior vena cava; RA, right atrium

insufflation was initiated and two 5-mm working ports were inserted in the left and right subcostal area. The central tendon of the diaphragm was identified and incised horizontally $(3-4 \mathrm{~cm})$ with a harmonic scalpel (Ethicon, Cincinnati, Ohio, United States). The pericardium was subsequently entered, and the laparoscopic ports removed. A cannula designed for pericardioscopic access (nContact Surgical, Morrisville, North Carolina, United States) was then placed inside the pericardial sac. The cannula with an endoscope was pushed into the oblique sinus to visualize the posterior wall of the LA and PVs. An irrigated, unipolar radiofrequency ablation device (VisiTrax nContact Surgical, Morrisville, North Carolina, United States) was passed through the cannula. Linear lesions (90 seconds each, $30 \mathrm{~W}$ power) were created.

Due to advancements in the surgical technique and our understanding of the AF pathology (LA fibrosis), the lesion pattern evolved over time. Patients with paroxysmal or persistent AF were more likely to receive lesions around the PVs ostia and linear applications at the mitral isthmus, LA roof, and on the posterior wall between inferior PVs. In contrast, patients with long-standing persistent $\mathrm{AF}$ were treated more aggressively with additional full posterior wall homogenization (FIGURE 1). All applications on the posterior wall of the LA were performed under fluoroscopic guidance to visualize the relationship between the ablating electrode and the esophagus. Temperature probes were placed to match the position of the ablating electrode. A $50-\mathrm{ml}$ sample of $30{ }^{\circ} \mathrm{C} 0.9 \%$ saline was injected into the pericardium prior to radiofrequency energy application to submerge the ablating electrode and reduce temperature spread. After creating all lesions, a small drain was placed behind the LA and passed through one of the 5-mm endoscopic ports. The midline fascia was closed with interrupted permanent sutures. Skin and port incisions were closed using absorbable sutures. The patients were discharged and readmitted for the second endocardial ablation after a minimum of 2 weeks.

Percutaneous endocardial catheter ablation protocol Endocardial ablation procedures were performed using the Seldinger technique to introduce 3 sheaths via the peripheral veins. A 10-polar electrode was introduced into the coronary sinus and a 4-polar electrode into the right ventricular apex. Subsequently, the Brockenbrough needle and the transseptal sheath (Mullins-type or steerable Agillis sheath, Abbot) were positioned in the upper right atrium, and the transseptal puncture was performed under the guidance of intracardiac pressures recorded from the tip of the needle. Immediately after the puncture, a single bolus of $10000 \mathrm{U}$ of heparin was administered, and an $8 \mathrm{~F}$ NaviStar (after market release, also Smarttouch and Smarttouch SF) irrigated-tip ablation electrode 
(Biosense Webster, Diamond Bar, California, United States) was introduced into the LA. Using an electroanatomical mapping system (CARTO, Biosense Webster), the isopotential map of the LA was created during sinus rhythm. A scar was defined as an area with an amplitude of bipolar atrial electrogram of less than $0.2 \mathrm{mV}$ and with electrical nonexcitability, and low-voltage area was defined as an area with an amplitude of bipolar electrogram between 0.2 and $0.5 \mathrm{mV}$. Once identified and marked on the map, low-voltage areas with persistent conductivity were subsequently ablated using radiofrequency applications, limiting the power to $40 \mathrm{~W}$ and application time to 60 seconds. We performed additional endocardial ablation if surgical lines were incomplete to prevent macroreentrant tachycardia, or if AF / atrial flutter (AFL) / atrial tachycardia persisted or were inducible, or in case of documented typical AFL. In 2 cases, cryoablation of PVs ostia was performed. Finally, electrical isolation of the veins (exit and entrance block), as well as additional ablation lines, was verified by stimulation maneuvers.

Efficacy and safety endpoints The primary endpoint was the recurrence of AF / AFL/atrial tachycardia documented on electrocardiogram, Holter monitoring (episode lasting more than 30 seconds), or an event recorder during a 12-month follow-up, excluding recurrences occurring in the first 90 days (blanking period). The patients were seen in an outpatient clinic and had at least 24-hour Holter monitoring at 3, 6, 9, and 12 months after the procedure. In 5 patients, electrocardiogram Loop Monitors (Reveal XT) was implanted at the time of the epicardial ablation procedure and controlled every 3 months. In 100 patients, 7-day Holter monitoring was performed at 6- and 12-month visits.

The secondary endpoints were periprocedural and long-term safety and adverse events during the follow-up, including death, stroke, rehospitalization due to any cause or AF / AFL (International Statistical Classification of Diseases, Tenth Revision [ICD-10] code I48), and need for ablation or cardioversion (either electrical or pharmacological).

Periprocedural safety was defined as the occurrence of major adverse cardiac events, such as death, myocardial infarction, stroke, major bleeding, or any procedure-related life-threatening event that occurred during the procedure or in-hospital period following the procedure, as defined by the 2017 expert consensus statement on catheter and surgical ablation of AF. ${ }^{24}$

The long-term analysis was performed based on data from the SILCARD (Cardiovascular Diseases in the Silesian Region in Poland; ClinicalTrials.gov identifier: NCT02743533) database, which enrolled all consecutive Silesian adult patients hospitalized in the cardiology, cardiac surgery, vascular surgery, or diabetology units for any reason, or hospitalized in the internal medicine or intensive care units with the principal diagnosis of cardiovascular disease or with the diagnosis of stroke at the neurology department.

The data provider for the SILCARD database was the Polish National Health Fund (Narodowy Fundusz Zdrowia), the only healthcare provider in Poland. The database was searched for a period of 3 years before surgical ablation and 3 years after completing hybrid ablation for events: death, stroke, rehospitalization for cardiac cardioversion (International Classification of Diseases, Ninth Revision [ICD-9] codes: 99.61, 99.62, 99.622, 99.624), re-ablation (ICD-9 codes: 37.341, 37.342), or bleeding complications (ICD-10 codes: I84.x, I85.0, K22.6; or ICD-9 blood transfusion $99.0 \times$ code); and admission for any cause and with principal diagnose of AF or AFL (ICD-10 code: 148 ). To assess the impact of HABL on the occurrence of these events, we compared a 3-year period before the first stage of ablation and a 3-year period after discharge after the second step of HABL. In cases where the endocardial step was not performed, the follow-up period started at discharge after surgical ablation.

Statistical analysis The distribution of variables describing patient and procedural characteristics were presented as mean (SD) or median and interquartile range (IQR) for continuous variables, and as the number of patients with percentages for categorical variables. For the survival analysis, Kaplan-Meier curves were plotted with the corresponding $P$ value of the log-rank or multivariate log-rank test (depending on the number of groups). Since data were not normally distributed (according to the Shapiro-Wilk test), the event rates per patient were compared with the Wilcoxon signed-rank test. The analysis was performed using the $\mathrm{R}$ and Python statistical packages, and a $P$ value of less than 0.05 was considered significant.

RESULTS Clinical characteristics The baseline characteristics are summarized in TABLE 1. The mean (SD) age was 51.02 (10.67) years; $74.7 \%$ of patients were men. The median (IQR) $\mathrm{CHA}_{2} \mathrm{DS}_{2}$-VASc score was 2 (1-2), and the median (IQR) HAS-BLED score was 1 (0-1). The majority of patients $(61.4 \%)$ had persistent AF $(23.4 \%$ with the long-standing variant), and 104 (65.8\%) presented with AF on admission. In total, 67 patients were previously ablated for AF, among them: 57 (85.1\%) with radiofrequency energy, 10 (14.9\%) with cryoablation, and 10 (14.9\%) with both radiofrequency and cryoablation. Eleven patients previously underwent cavotricuspid isthmus (CTI) ablation for typical AFL only. 
TABLE 1 Patient characteristics

\begin{tabular}{|c|c|c|}
\hline \multicolumn{2}{|l|}{ Variable } & All patients $(n=158)$ \\
\hline \multicolumn{2}{|c|}{ Age, y, mean (SD) } & $51(10.7)$ \\
\hline \multicolumn{2}{|l|}{ Male sex } & $118(74.7)$ \\
\hline \multirow[t]{2}{*}{ Type of AF } & Paroxysmal & $61(38.6)$ \\
\hline & Persistent/long-term persistent & $97(61.4) / 37(23.4)$ \\
\hline \multicolumn{2}{|c|}{ Previous failed catheter ablation for $\mathrm{AF}$} & $66(41.8)$ \\
\hline \multicolumn{2}{|c|}{ History of heart failure } & $58(36.7)$ \\
\hline \multicolumn{2}{|c|}{ Tachycardia-induced cardiomyopathy } & $30(19)$ \\
\hline \multicolumn{2}{|c|}{ Hypertension } & $80(50.6)$ \\
\hline \multicolumn{2}{|c|}{ Diabetes mellitus } & $22(13.9)$ \\
\hline \multicolumn{2}{|c|}{ Coronary artery disease } & $22(13.9)$ \\
\hline \multicolumn{2}{|c|}{ Previous myocardial infarction } & $10(6.3)$ \\
\hline \multicolumn{2}{|c|}{ Stroke or TIA } & $13(8.2)$ \\
\hline \multicolumn{2}{|c|}{ History of smoking } & $88(55.7)$ \\
\hline \multicolumn{2}{|c|}{ Hyperlipidemia } & $83(52.5)$ \\
\hline \multicolumn{2}{|c|}{ History of thyroid disorders (euthyroidism at HABL) } & $10(6.3)$ \\
\hline \multicolumn{2}{|c|}{ Chronic kidney disease } & $8(5)$ \\
\hline \multicolumn{2}{|c|}{ Pacemaker / ICD implanted } & $14(8.8)$ \\
\hline \multicolumn{2}{|c|}{$\mathrm{CHA}_{2} \mathrm{DS}_{2}$-VASc ${ }^{\mathrm{a}}$ score, median (IQR) } & $2(1-2)$ \\
\hline \multicolumn{2}{|c|}{ HAS-BLED ${ }^{\mathrm{b}}$ score, median (IQR) } & $1(0-1)$ \\
\hline \multicolumn{2}{|c|}{ BMI, kg/m², mean (SD) } & $29.15(3.8)$ \\
\hline \multicolumn{2}{|c|}{ LVEF, \%, mean (SD) } & $51(10.7)$ \\
\hline \multicolumn{2}{|c|}{ LA size, mm, mean (SD) } & $44.4(6.7)$ \\
\hline \multicolumn{2}{|c|}{ AF on admission } & $104(65.8)$ \\
\hline
\end{tabular}

Data are presented as number (percentage) of patients unless otherwise indicated.

Abbreviations: AF, atrial fibrillation; BMI, body mass index; HABL, hybrid ablation; ICD, implantable cardioverter defibrillator; LA, left atrial; LVEF, left ventricular ejection fraction; TIA, transient ischemic attack

In 58 patients (36.7\%), heart failure was diagnosed, in 30 (19\%), it was related to tachycardia-induced cardiomyopathy, defined as left ventricular ejection fraction of $35 \%$ or less due to long-lasting arrhythmia. The median (IQR) exercise tolerance in the study population assessed on the New York Heart Association Functional Classification scale was 2 (2-2) class.

Procedural data The procedural data are summarized in TABLE 2. In all 158 patients, the minimally invasive surgical procedure was performed with a median (IQR) in-hospital stay of 3 (2-4) days.

The patients were discharged and readmitted for the second endocardial ablation after a minimum of 2 weeks (median [IQR], 51 [24.4-128.5] days). After clinical evaluation, no endocardial ablation was performed in 23 cases (14.55\%), including 19 patients who showed significant clinical improvement without any AF episode in follow-up (after previous failed endocardial ablation), 2 patients who had surgical complications (1 with constrictive pericarditis and 1 with significant PV stenosis but no AF recurrence), and 2 patients with left atrial appendage thrombus (a contraindication for ablation).

The extent of endocardial ablation In 135 patients who underwent endocardial ablation, 49 (36.3\%) presented with AF / AFL by admission. In 2 cases, cryoablation of the PV ostia was accomplished. In the remaining 133 patients, endocardial mapping using 3-dimensional electroanatomical system showed low-voltage and scar areas within the LA in all patients, mostly (94.7\%) in the LA posterior wall (TABLE2). In 7 patients, all PVs were completely isolated after surgical ablation, and $34 \%$ of patients required only the completion of ablation lines around PVs. More than half of patients required additional substrate modification on the posterior wall to eliminate 
TABLE 2 Procedural and in-hospital data of the study patients

ICU postoperative hospitalization length, $d$, median (IQR)

$2(1-2)$

Cardiac surgery hospitalization length, $d$, median (IQR)

$3(2-4)$

Low voltage and scar areas identified with mapping $(n=133)$

Posterior wall

$126(94.7)$

Mitral isthmus

$42(31.6)$

Septal substrate

$19(14.3)$

Range of endocardial ablation $(n=135)$

PVI only

$46(34)$

LA posterior wall isolation Any complementation

\begin{tabular}{ll} 
Roof line only \\
\cline { 2 - 2 } & "Box" lines (roof + between inferior PVs) \\
\hline
\end{tabular}

Cavotricuspid isthmus

$71(52.6)$

\begin{tabular}{ll}
\hline Cavotricuspid isthmus & $32(23)$ \\
\hline Mitral isthmus line & $17(12.6)$ \\
\hline Coronary sinus ablation & $31(23)$ \\
\hline Ectopic atrial tachycardia in right atrium & $2(1.5)$ \\
\hline AVNRT & $1(0.74)$ \\
\hline Medication at discharge after hybrid ablation & $1(0.74)$ \\
\hline VKA/NOAC & \\
\hline MRA & $105(66.5) / 53(33.5)$ \\
\hline ACEI/ARB & $67(42.4)$ \\
\hline Statins & $83(52.5)$ \\
\hline B-Blockers & $72(45.6)$ \\
\hline Propafenone & $124(78.5)$ \\
\hline Sotalol & $54(34.4)$ \\
\hline Amiodarone & $28(17.7)$ \\
\hline Digoxin & $28(17.7)$ \\
\hline Dronedarone & $5(3.2)$ \\
\hline Calcium channel blocker & $1(0.6)$ \\
\hline
\end{tabular}

Data are presented as number (percentage) of patients unless otherwise indicated.

Abbreviations: ACEI, angiotensin-converting-enzyme inhibitors; ARB, angiotensin-receptor blockers; AVNRT, atrioventricular nodal reentry tachycardia; ICU, intensive care unit; MRA, mineralocorticoid receptor antagonists; NOAC, non-vitamin K antagonist; PVI, pulmonary vein isolation; VKA, vitamin K antagonists; others, see TABLE1

slow-conducting areas within low-voltage areas and to isolate the LA posterior wall. The mitral isthmus was ablated in 31 cases (23\%), the septal line in 17 (12.6\%), and additionally, the CTI in 32 (23\%). In 2 cases, right atrial arrhythmia was induced (ectopic atrial tachycardia, atrioventricular nodal reentry tachycardia) and ablated.

The majority $(78 \%)$ of patients were discharged with a prescription of $\beta$-blockers and nearly one-third, with propafenone (tABLE 2). Twelve patients required additional electrical cardioversion due to AF before discharge.
Safety During the study, serious adverse events were reported in the perioperative period in $7 \mathrm{pa}-$ tients after surgical ablation, and in 10 patients after endocardial ablation (TABLE 3). The major AE (ie, death of unknown cause, bleeding requiring sternotomy, cardiac tamponade, and transient ischemic attack) have been described in detail and discussed in our preliminary report. ${ }^{8}$ Two additional transient ischemic attacks were observed between 24 and 48 hours after ablation, only in cases with anticoagulation bridged with heparin, but they resolved quickly after administration 


\begin{tabular}{|c|c|c|c|}
\hline Variable & All patients & $\begin{array}{l}\text { HABL as } \\
\text { the first-time } \\
\text { ablation }\end{array}$ & $\begin{array}{l}\text { HABL after } \\
\text { previous } \\
\text { ablations }\end{array}$ \\
\hline \multicolumn{4}{|l|}{ Surgical complications $(n=158)$} \\
\hline Any & 7 & - & - \\
\hline Bleeding including tamponade and blood transfusion & 1 & 1 & 0 \\
\hline Re-operation & 0 & 0 & 0 \\
\hline Pericardial and pleural effusion & 0 & 0 & 0 \\
\hline Stroke/TIA & $0 / 1$ & 0 & $0 / 1$ \\
\hline Death & 1 & 0 & 1 \\
\hline PVI stenosis & 2 & 0 & 2 \\
\hline Pericarditis (constrictive) & 1 & 1 & 0 \\
\hline Transient phrenic nerve palsy & 1 & 0 & 1 \\
\hline \multicolumn{4}{|l|}{ Endocardial ablation complications $(n=135)$} \\
\hline Any & 10 & - & - \\
\hline Bleeding requiring blood transfusion & 0 & 0 & 0 \\
\hline Minor bleeding (puncture site) & 2 & 1 & 1 \\
\hline AV fistula (puncture site) & 1 & 1 & 0 \\
\hline Pericardial and pleural effusion / tamponade (sternotomy) & $3 / 1$ & $2 / 1$ & 1 \\
\hline Stroke/TIA & $0 / 2$ & $0 / 1$ & $0 / 1$ \\
\hline Other (acute ischemia anesthesia related) & 1 & 1 & 0 \\
\hline Death & 0 & 0 & 0 \\
\hline
\end{tabular}

Data are presented as number of patients.

Abbreviations: see TABLE 1

of non-vitamin $\mathrm{K}$ antagonists. In one patient, a transient right forearm ischemic episode occurred due to erroneous vein puncture and local administration of anesthetic drugs. In 2 cases, we observed significant PVs stenoses on a CT scan, which were fortunately asymptomatic for the patients; subsequent surgical ablations were performed at an increased distance from the PV ostia. We did not observe more periprocedural complications depending on the history of failed previous catheter ablation procedures. It is worth noting that all most serious complications occurred during the implementation of the hybrid method (before patient number 15 in our center).

Arrhythmic efficacy One-year follow-up data were available for 115 patients at our center. The recurrence of AF was noted in 7 patients after a 3-month blanking period, and in 20, 23, and 25 patients after a 6-, 9-, and 12-month follow-up, respectively. We did not observe significant differences in the risk of $\mathrm{AF}$ recurrence while comparing different types of AF. Similarly, the results in patients ablated for the first time and after previous failed catheter procedures were comparable (FIGURE2).
Long-term follow-up Comparison of 3 years period: pre-and post-hybrid ablation We compared the data of 110 patients who completed a 3-year follow-up (and 146 patients after 1-year follow-up) after HABL based on the SILICARD database: 41 with paroxysmal, 36 with persistent, and 33 with long-standing persistent AF. During this period, 3 patients died: one died 2 days after ablation (discussed in our previous report), ${ }^{8}$ one died 28 days after ablation with a diagnosis of fatal stroke, and one died due to an unknown cause 924 days after the ablation.

We found no differences in the occurrence of stroke (1 vs 1 patient) or bleeding episodes requiring medical contact (4 vs 4 patients) in a 3-year period before the first stage of ablation compared to a 3-year period after discharge after the second step of hybrid ablation. There was a significant reduction in the number of hospitalizations for AF as well as for any cause, need for re-ablation or cardioversions (FIGURES 3 and 4). We observed the same tendency irrespective of the type of AF, except the need for any cause hospitalization in long-standing persistent AF; ablation in persistent variant of $\mathrm{AF}$ or cardioversion in patients with paroxysmal or 


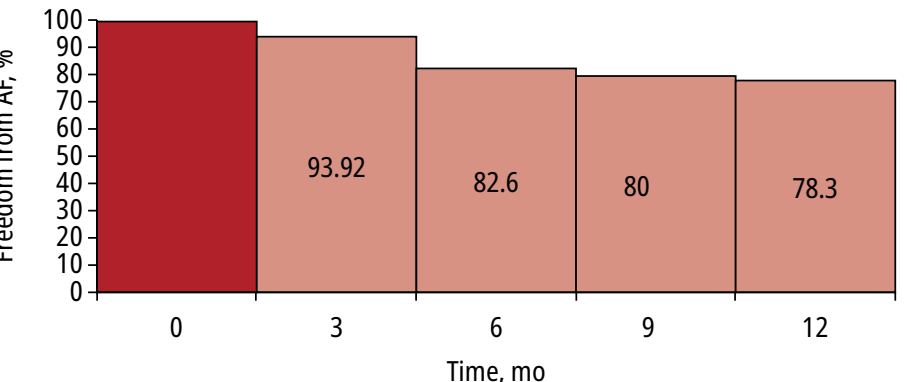

B

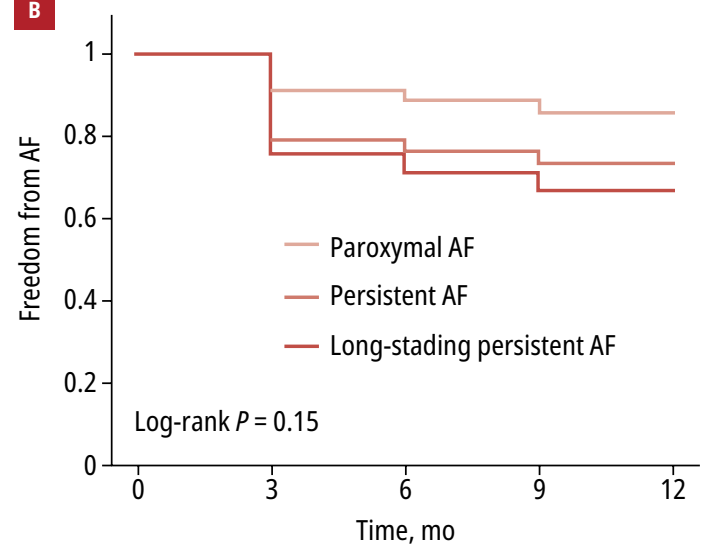

Paroxymal AF At risk 62

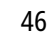

37

28

Persistent AF
46

43

29 At risk 59

Long-stading persistent AF At risk 37

30

26

FIGURE 2 Freedom from atrial fibrillation after hybrid ablation in a 12-month follow-up for all patients (A), according to the type of atrial fibrillation (B) and hybrid approach (HABL) as the first ablation attempt or procedure after previous failed catheter ablation (C)

Abbreviations: see TABLE 1

33

23

At risk 67

$17 \quad 16 \quad 14$

14

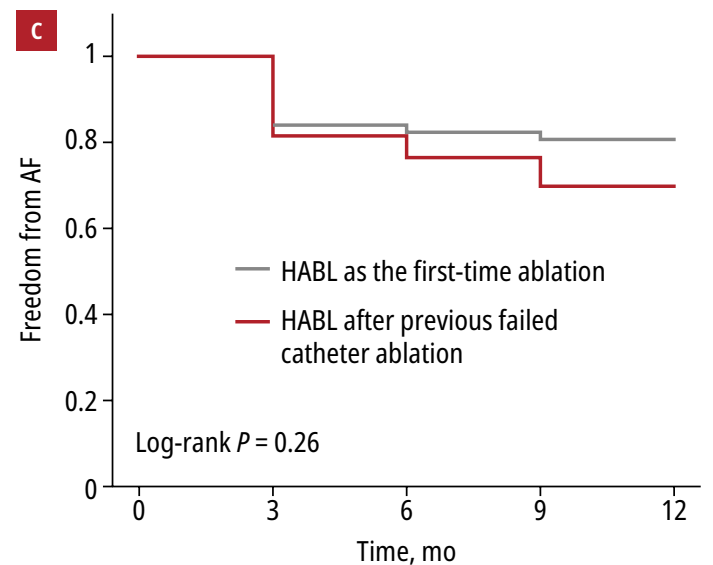

First-time ablation At risk 91
75

43 persistent AF (FIGURE 5). However, ablation frequency in the whole group did not differ at 12 months before and after HABL.

DISCUSSION The hybrid ablation approach, which combines the advantages of epicardial and endocardial therapy, allows for achieving not only patient-tailored treatment but also transmural and durable lesions. It also helps avoid restrictions resulting from the risk of damage to the esophagus during extensive endocardial ablation on the posterior LA wall or lack of assessment of electrical activity in the surgical approach. Based on the previous histological and animal studies, the nonhomogeneous myocardial arrangement of the LA posterior wall with conduction anisotropy between epiand endocardial layers predisposes to the presence micro-reentry and focal arrhythmias being the most common arrhythmia source next to PVs. ${ }^{25,26}$ A hybrid approach allows effective ablation (homogenization) of the LA posterior wall, which is especially important in persistent variants of AF. Postponing the second (endocardial) ablation allows surgical lesion maturation without negatively influencing long-term results. ${ }^{27}$

An essential electrophysiological observation from our study is that surgical ablation alone is not sufficient for the complete isolation of low amplitude and slow conduction areas in most patients. This observation is in line with previously published studies ${ }^{28,29}$; however, so far, no data were published regarding the range of lesions after ablation using a minimally invasive, transabdominal approach. Compared with other types of access, we found that this approach was not inferior in terms of the possible extent of the ablation. In our study, only one-third of patients underwent point supplementation of ablative lines around PVs; the remaining cohort required more extensive modification of the substrate to prevent the recurrence of arrhythmia and the formation of potential re-entry loops. Therefore, we presume that to increase the effectiveness of the procedure and for individualized treatment, accurate voltage mapping 


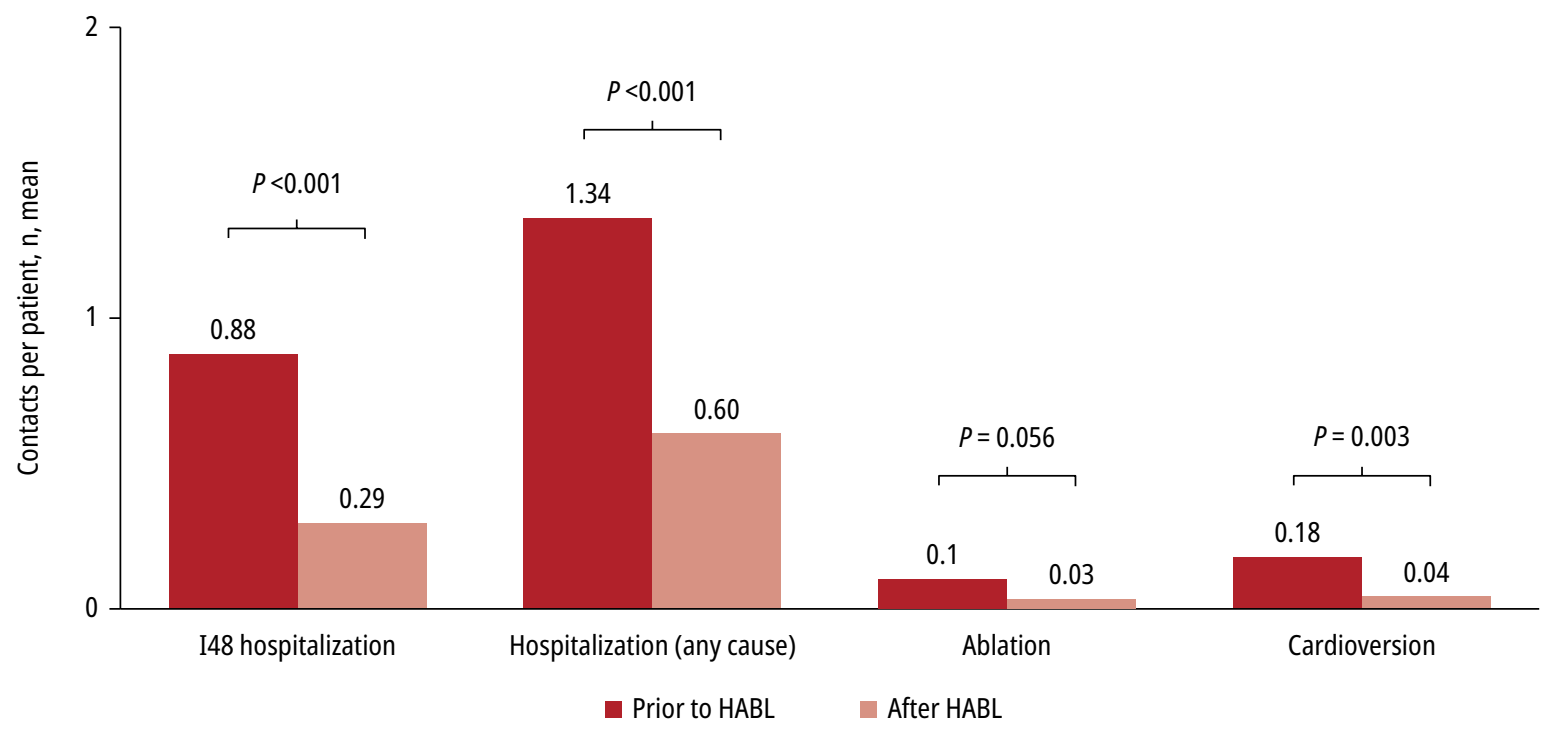

FIGURE 3 Comparison of medical contacts in the 12 months prior and 12 months after hybrid ablation (HABL) of atrial fibrillation ( $n=146)$ : hospitalization with diagnosis of atrial fibrillation or flutter (ICD-10 code I48), any cause hospitalization, ablation or cardioversion

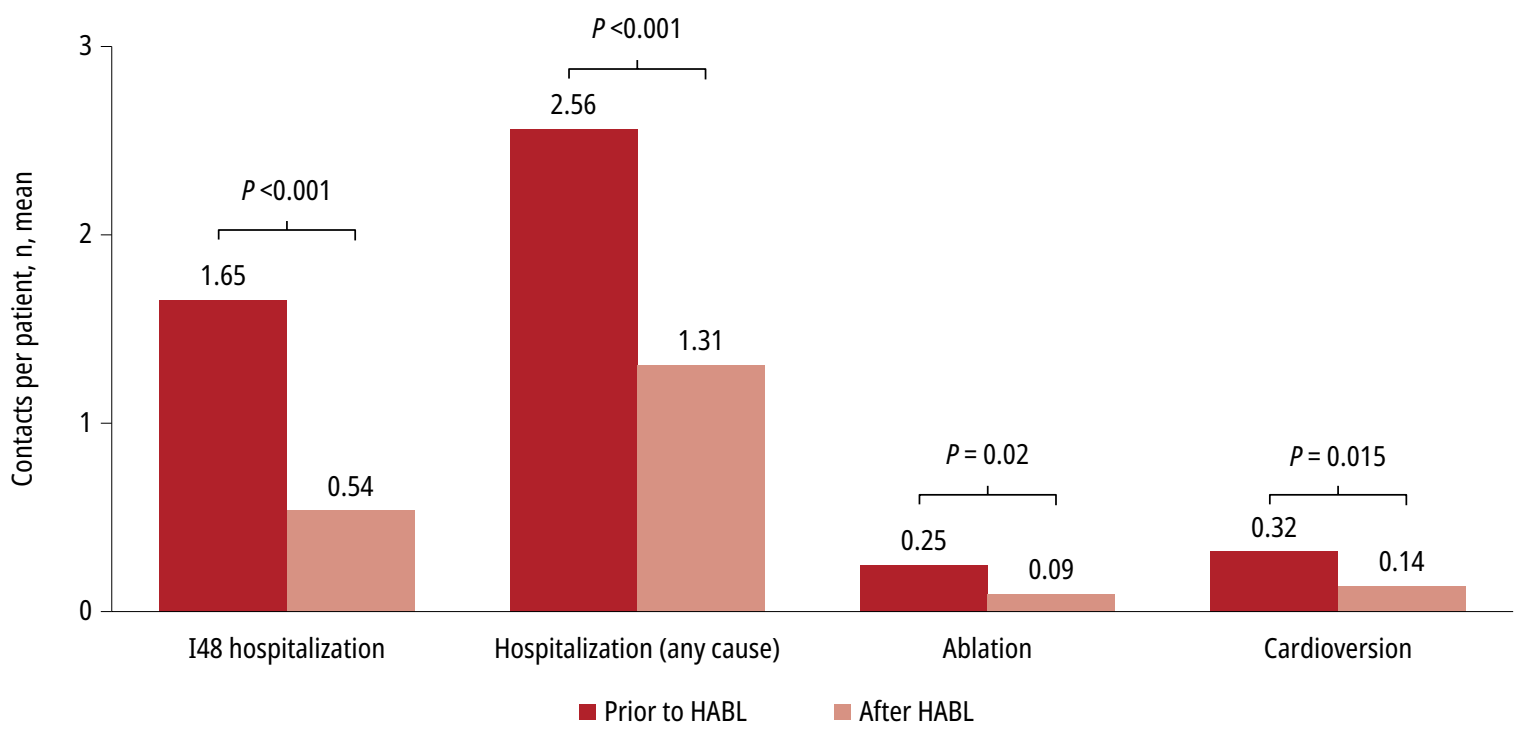

FIGURE 4 Comparison of medical contacts in the 3 years prior and 3 years after hybrid ablation (HABL) of atrial fibrillation ( $n=110)$ : hospitalization with diagnosis of atrial fibrillation or flutter (ICD-10 code I48), any cause hospitalization, ablation or cardioversion

during the second step of the hybrid approach is not only reasonable but also necessary for proper tailored therapy. Nowadays, dedicated multielectrode mapping catheters facilitate fast and accurate creation of high-resolution maps based on thousands of electroanatomical points. However, due to long duration of our study, most of the maps were created using available at that time ablations and spiral (lasso) catheters. It is worth noting that some patients (after previous unsuccessful endocardial ablation) did not have any recurrence of arrhythmia after epicardial ablation only and did not need another endocardial ablation procedure. It supports the theory of the relevance of posterior wall isolation to prevent $\mathrm{AF}$ recurrence.
As AF invasive treatment is an elective procedure, there are special concerns regarding procedural safety. At our center, most serious AEs occurred during the first hybrid ablations, which was likely due to the initial lack of local experience and learning curve, as observed in other centers (ie, the initial risk was higher and diminished with practice). ${ }^{16,27,30-32}$ After gaining experience, the AEs became incidental and mostly related to periprocedural proceeding. The most common serious AE was transient ischemic attack, which occurred on the next day after ablation during restarting oral anticoagulation by insufficient heparin bridging. However, as the procedural protocol evolved, and nowadays, endocardial ablation is recommended without 


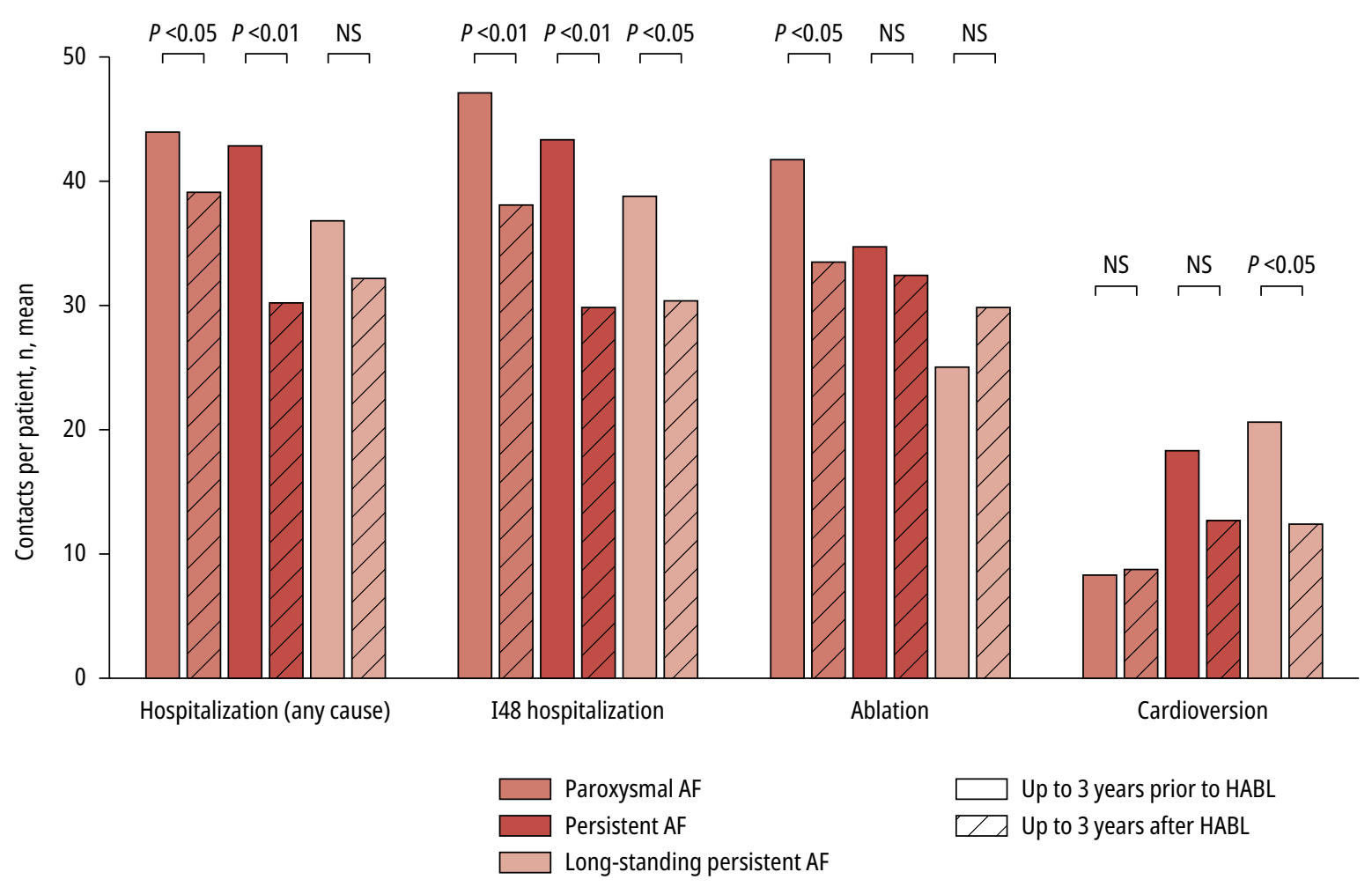

FIGURE 5 Comparison of medical contacts in the 3 years prior and 3 years after hybrid ablation (HABL) of atrial fibrillation $(n=110)$ : hospitalization with diagnosis of atrial fibrillation or flutter (ICD-10 code I48), any cause hospitalization, ablation, or cardioversion—-depending on the type of atrial fibrillation Abbreviations: LSPAF, long-standing persistent atrial fibrillation; PAF, paroxysmal atrial fibrillation; PSAF, persistent atrial fibrillation

interrupting of oral anticoagulation, we expect further risk reduction of this complication.

The long-term results observed provide new hope that the hybrid approach can improve outcomes in challenging patients with AF, especially after ineffective percutaneous ablation. We found no arrhythmia recurrence in nearly $80 \%$ of patients, comparable to other convergent procedure reports..$^{16-19}$ Moreover, a low recurrence rate 6 months after the procedure may indicate the durability of created lesions and the constancy of treatment results. Similar findings, but with a videothoracoscopy technique and follow-up extended to 3 years, have been reported by Maesen et al. ${ }^{17}$ The long-term results in our follow-up are satisfying, even though a significant number of patients had heart failure-most often associated with a significant structural remodeling of LA limiting the effectiveness of therapy.

According to previous research and metanalyses, the hybrid approach is characterized by better efficacy in patients with persistent AF compared with repeated percutaneous ablation. ${ }^{32}$ A novelty of our work is the long-term comparison of the period before and after hybrid treatment, which shows a significant reduction in the need for rehospitalizations (especially due to $\mathrm{AF}$ ), repeated ablations, or cardioversions. These results not only confirm the long-term effectiveness of the therapy but also highlight its positive effect on reducing the burden on the healthcare system.
In our opinion, hybrid ablation should be considered a therapeutic option in patients with an extensive arrhythmic substrate and after failed percutaneous ablations. We believe our observations will help in appropriate preparation and implementation of hybrid ablation in new centers.

Limitations There are certain limitations to the study. First of all, it is a retrospective analysis with data collected from patient's records. Second, the arrhythmic follow-up was limited only to 12 months and mostly based on Holter monitoring records. Nowadays, the available loop-recorders provide more reliable data. Third, the number of hospitalization diagnoses reported to the Polish National Health Found system is limited. However, we believe that in cases of hospitalization due to symptomatic AF, given the need for cardioversion or reablation, the diagnosis of I48 was always reported. Fourth, this analysis includes a limited number of patients. Even though this is, to the best of our knowledge, one of the largest cohorts investigated with regard to the topic, and the comparison of medical contacts is unique.

Conclusions The hybrid multidisciplinary approach to treatment of AF is a safe and, in long-term follow-up, very effective therapy which reduces the healthcare burden and could be considered as an alternative therapeutic option especially in patients with persistent AF. 


\section{ARTICLE INFORMATION}

CONFLICT OF INTEREST PB and MOZ are consultants at AtriCure Europe B.V. Other authors declare no conflict of interest.

OPEN ACCESS This is an Open Access article distributed under the terms of the Creative Commons Attribution-Non Commercial-No Derivatives $4.0 \mathrm{In}$ ternational License (CC BY-NC-ND 4.0), allowing third parties to download articles and share them with others, provided the original work is properly cited not changed in any way, distributed under the same license, and used for noncommercial purposes only. For commercial use, please contact the journal office at kardiologiapolska@ptkardio.pl.

HOW TO CITE Buchta P, Sierpiński R, Myrda K, et al. New hope for patients and challenges for the multidisciplinary arrhythmia team: a hybrid convergent approach for atrial fibrillation treatment. Kardiol Pol. 2020; 78: 1243-1253. doi:10.33963/KP.15640

\section{REFERENCES}

1 Mairesse GH, Moran P, Van Gelder IC, et al. Screening for atrial fibrillation: a European Heart Rhythm Association 1. (EHRA) consensus document endorsed by the Heart Rhythm Society (HRS), Asia Pacific Heart Rhythm Society (APHRS), and Sociedad Latinoamerica na de Estimulacion Cardiaca y Electrofisiologia (SOLAECE). Europace. 2017; 19: 1589-1623.

2 Kirchhof P, Benussi S, Kotecha D, et al. 2016 ESC guidelines for the management of atrial fibrillation developed in collaboration with EACTS. Europace. 2016; 18: 1609-1678.

3 Calkins H, Hindricks G, Cappato R, et al. 2017 HRS/EHRA/ECAS/APHRS/SOLAECE expert consensus statement on catheter and surgical ablation of atrial fibrillation. Heart Rhythm. 2017; 14: e275-e444.

4 Morillo CA, Klein GJ, Jones DL, Guiraudon CM. Chronic rapid atrial pacing. Structural, functional, and electrophysiological characteristics. of a new model of sustained atrial fibrillation. Circulation. 1995; 91: 1588-1595.

5 Wakili R, Voigt N, Kääb S, et al. Recent advances in the molecular pathophysiology of atrial fibrillation. J Clin Invest. 2011; 121: 2955-2968.

6 Estes III NA, Sacco RL, Al-Khatib SM, et al. American Heart Association atrial fibrillation research summit: a conference report from the American Heart Association. Circulation. 2011; 124: 363-372.

7 Schotten U, Verheule S, Kirchof P, Goette A. Pathophysiological mechanisms of atrial fibrillation: a translational appraisal. Physiol Rev. 2011; 91: 265-325.

8 Yang F, Tiano J, Mittal S, et al. Towards a mechanistic understanding and treatment of a progressive disease: atrial fibrillation. J Atr Fibrillation. 2017; 10: 1627.

9 Iwasaki Y, Nishida K, Kato T, Nattel S. Atrial fibrillation pathophysiology implications for management. Circulation. 2011; 124: 2264-2274.

10 Kiedrowicz R, Wielusinski M, Wojtarowicz A, Kazmierczak J. Predictors of the voltage derived left atrial fibrosis in patients with long-standing persistent atrial fibrillation. Cardiol J. 2020 May 18. [Epub ahead of print].

11 Masuda M, Fujita M, Iida 0 , et al. Influence of underlying substrate on atrial tachyarrhythmias after pulmonary vein isolation. Heart Rhythm. 2015; 13: 870-878.

12 Rolf S, Kircher S, Arya A, et al. Tailored atrial substrate modification based on low-voltage areas in catheter ablation of atrial fibrillation. Circ Arrhythm Electrophysiol. 2014; 7: 825-833.

13 Kowalski M, Grimes MM, Perez FJ, et al. Histopathologic characterization of chronic rediofrequency ablation lesions for pulmonary vein isolation. J Am Coll Cardiol. 2012; 59: 930-938.

14 Piorkowski C, Kronborg M, Hourdain J, et al. Endo-/epicardial catheter ablation of atrial fibrillation. Feasibility, outcome, and insights into arrhythmia mechanisms. Circulation: Arrhythm Electrophysiol. 2018; 11: e005748.

15 Glover BM, Hong KL, Baranchuk A, et al. Preserved left atrial epicardial conduction in regions of endocardial "isolation". JACC Clin Electrophysiol. 2018; 4: 557-558.

16 Vroomen M, Pison L. Hybrid ablation for atrial fibrillation: a systematic review. J Interv Card Electrophysiol. 2016; 47: 265-274.

17 Xiaokang L, Li B, Zhang D, et al. Efficacy and safety of the convergent atrial fibrillation procedure: a meta-analysis of observational studies. Interact Cardiovasc and Thorac Surg. 2019; 28: 169-176.

18 Pison L, La Meir M, van Opstal J, et al. Hybrid thoracoscopic surgical and transvenous catheter ablation of atrial fibrillation. J Am Coll Cardiol. 2012; 60: 54-61.

19 Maesen B, Pison L, Vroomen M, et al. Three-year follow-up of hybrid ablation for atrial fibrillation. Eur J Cardiothorac Surg. 2018; 53 (suppl): i26-i32.

20 Matevž, J, Žižek D, Geršak ŽM, Geršak B. Comparison of treatment outcomes between convergent procedure and catheter ablation for paroxysmal atrial fibrillation evaluated with implantable loop recorder monitoring. J Cardiovasc Electrophysiol. 2018; 29: 1073-1080.

21 Zembala M, Filipiak K, Kowalski 0, et al. Minimally invasive hybrid ablation procedure for the treatment of persistent atrial fibrillation: one year results. Kardiol Pol. 2012; 70: 819-828.

22 Kalarus Z, Zembala M, Kowalski 0, et al. Minimally invasive, hybrid ablation in a patient with persistent atrial fibrillation - early experience. Kardiol Pol. 2009; 67: 1057-1062.

23 Zembala M, Filipiak K, Kowalski 0, et al. Staged hybrid ablation for persisten and longstanding persistent atrial fibrillation effectively restores sinus rhythm in long-term observation. Arch Med Sci. 2017; 13: 109-117.
24 Calkins H, Hindricks G, Cappato R, et al. 2017 HRS/EHRA/ECAS/APHRS/SO LAECE expert consensus statement on catheter and surgical ablation of atrial fibrillation. Europace. 2018; 20: e1-e160.

25 van der Does L, Knops $P$, Teuwen $C$, et al. Unipolar atrial electrogram morphology from an epicardial and endocardial perspective. Heart Rhythm. 2018; 15: 879-887.

26 Eckstein J, Maesen B, Linz D et al. Time course and mechanisms of endo-epicardial electrical dissociation during atrial fibrillation in the goat. Cardiovasc Res. 2011; 89: 816-824.

27 Bulava A, Mokracek A, Hanis J. Sequential hybrid procedure for persistent atrial fibrillation. J Am Heart Assoc. 2015; 4: e001754

28 Krul S, Pison L, La Meir M, et al. Epicardial and endocardial electrophysiological guided thoracoscopic surgery for atrial fibrillation: a multidisciplinary approach of atrial fibrillation ablation in challenging patients. Int I Cardiol. 2014; 173: 229-235.

29 Al-Jazairi.MIH, Rienstra M, Klinkenberg TJ. Hybrid atrial fibrillation ablation in patients with persistent atrial fibrillation or failed catheter ablation. Neth Heart J. 2019; 27: 142-151.

30 Geršak B, Zembala MO, Müller D, et al., European experience of the convergent atrial fibrillation procedure: multicenter outcomes in consecutive patients. J Thorac Cardiovasc Surg. 2014; 147: 1411-1416.

31 Syed FF, Oral H. Electrophysiological perspectives on hybrid ablation of atrial fibrillation. J Atr Fibrillation. 2015; 8: 1290.

32 van der Heijden CAJ, Vroomen M, Luermans JG, et al. Hybrid versus cathete ablation in patients with persistent and longstanding persistent atrial fibrillation: a systematic review and meta-analysis. Eur J Cardiothorac Surg. 2019: 3: 433-443. 33 Wojtaszczyk A, Buchta P, Myrda K, et al. Hybrid dual stage closed chest ablation of persistent atrial fibrillation. Cor et Vasa. 2017; 59: e337-e344. 